\title{
THE PHOTOGRAMMETRIC SURVEY METHODOLOGIES APPLIED TO LOW COST 3D VIRTUAL EXPLORATION IN MULTIDISCIPLINARY FIELD
}

\author{
C. Palestini, A. Basso \\ Dipartimento di Architettura, Università degli Studi “G. d'Annunzio” Pescara, Italy - \\ caterinapalestini@ libero.it, alessandro.basso77@gmail.com
}

Commission II

KEY WORDS: 3d, Interaction, photogrammetric survey, virtual empathy, virtual reality

\begin{abstract}
:
In recent years, an increase in international investment in hardware and software technology to support programs that adopt algorithms for photomodeling or data management from laser scanners significantly reduced the costs of operations in support of Augmented Reality and Virtual Reality, designed to generate real-time explorable digital environments integrated to virtual stereoscopic headset. The research analyzes transversal methodologies related to the acquisition of these technologies in order to intervene directly on the phenomenon of acquiring the current VR tools within a specific workflow, in light of any issues related to the intensive use of such devices, outlining a quick overview of the possible "virtual migration" phenomenon, assuming a possible integration with the new internet hyper-speed systems, capable of triggering a massive cyberspace colonization process that paradoxically would also affect the everyday life and more in general, on human space perception. The contribution aims at analyzing the application systems used for low cost $3 \mathrm{~d}$ photogrammetry by means of a precise pipeline, clarifying how a $3 \mathrm{~d}$ model is generated, automatically retopologized, textured by color painting or photo-cloning techniques, and optimized for parametric insertion on virtual exploration platforms. Workflow analysis will follow some case studies related to photomodeling, digital retopology and "virtual 3d transfer" of some small archaeological artifacts and an architectural compartment corresponding to the pronaus of Aurum, a building designed in the 1940s by Michelucci. All operations will be conducted on cheap or free licensed software that today offer almost the same performance as their paid counterparts, progressively improving in the data processing speed and management.
\end{abstract}

\section{INTRODUCTION}

The evolution of the spatial concept in the virtual sense coincides more and more with a substantial transformation of the perception of contemporary spaces, linked to factors specific to Information Technology. In the tendency that diffuses in a widespread and transversal way many of the attitudes that represent contemporary culture evolve an ephemeral landscape that tends to overlap the real one, identifying, in its infinite connotations, the dynamic interconnection of entities reconfigured in alternative existence. "Virtual migration", understood as progressive adherence to cyberspace and all its prerogatives, can now be viewed concretely in the light of the integration of virtual devices with the new hypnotic systems, capable of amplifying media diffusion and shared real-time experience, has undertaken a rapid diffusion process. The latter appears to be linked to several factors, including the actual social relational impossibilities of our times or the needs associated with the evolution of the logistics of professional fields, such as the medical or cultural-museum divulgation, or simply for motivational reasons dictated by the current desire to escape from reality. This scenario of concrete modification of behavioral trends in the daily and collective social setting has convinced financial sponsors and private investors to support hardware and software research in backing of virtual cloning aimed at transferring to virtual multimedia platforms. We therefore witness a multiplication of more and more user friendly programs that adopting algorithms acts to Photomodelling, or management of data from recovery systems Laser scanner, functional to generate a three-dimensional copy of elements directly cultured from reality and in functional parallel to interactive and virtual design, in order to generate easy real-time explorable digital environments (RTR) on ever

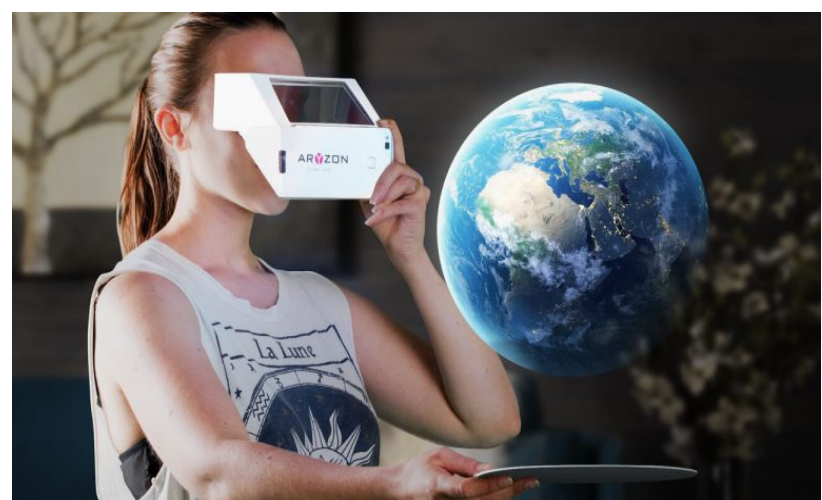

Figure 1. AR Lowcost Aryzon system, presentation image from https://aryzon.com/

widespread virtual platforms such as Unreal engine or Unity, integrated progressively with interactive hardware such as VR Homido V2 or Google Daydream View, optical viewers similar to the famous Oculus Rift, which can accommodate all kinds of iOS and Android smartphones. The increase in demand over the last five years has considerably lowered the cost of virtual applications thanks to the involvement of production companies from developing countries who have bet, driven by accurate market research, on the mass spread of such equipment, making them in fact to a clientele that is no longer specialized. The welcome to VR devices and all software that allows the creation of scenarios compatible with an interactive experience is much more appealing than the one reserved for other technologies, 
and the process of distributing such tools in different professional sectors seems faster compared to the past, thanks also to the influence derived from the collective imagination generated by the sci-fi subculture, fed by the seventies by countless examples of science fiction films and novels where the concept of virtual dimension, cyberspace and digital technoculture finished to contaminate expectations on the future to come. In this regard, it is essential, in the light of a process already underway, to outline the benefits and criticalities associated with the intensive use of such tools in order to improve their usability and expanding their use into more and more sophisticated domains. It is therefore possible to implement such instruments not only for entertainment but to be considered more active and useful in cultural, architectural, archaeological, military, medical and engineering fields. The perception of real content in a virtual dimension, more and more tangible, indistinguishable from physical reality, able to offer, thanks to highly photorealistic rendering algorithms, a replica, a clone of an architectural or archaeological environment exactly identical to the real one, favors the virtual reproduction of spaces that are not inaccessible, physically unexplored, as they are placed in areas that are hard to reach or simply no longer exist. As part of the debate on the strategies to be followed for expanding the use of digital models, the contribution aims at analyzing the application systems used for low cost $3 \mathrm{~d}$ photogrammetry by means of a precise pipeline, clarifying as a $3 \mathrm{~d}$ model of a architecture or archeological artifact can be generated, automatically retopologized in Zbrush, a relatively low cost program used mainly by digital artists, textured by color painting or photo-cloning techniques, and optimized for its parametric insertion on platform of real time virtual exploration (in our case Unreal Engine 4.15).

The paper proposes in the first phase the introduction and analysis of the adopted workflow, which includes several steps from the acquisition of photogrammetric data to the technical explanation of the programs used, followed by an analysis of some case studies related to a reconstruction $3 \mathrm{~d}$ of some small archaeological artefacts, up to the three-dimensional photogrammetric survey, digital remodeling and "virtual transfer" on RTR platform of a part of the Aurum complex in Pescara, a building designed by the architect Michelucci in the 1940 s on a pre-existence of the early twentieth century, the Kursaal with classical connotations. All operations will be conducted with low-cost or licensed software, tools that today offer almost the same performance as paid versions, progressively improving even in the speed of calculations and data management.

\section{INTERACTION, IMMERSION AND PRESENCE IN THE LOWCOST VIRTUAL ERA}

The reduction of production costs and consequently of the commercial product favored the gradual emergence of new three-dimensional data acquisition criteria and the subsequent transfer to immersive scenario editing platforms by the active use of HMD virtual devices, triggering an technical and instrumental upgrading process of the world of work, and in particular of architectural design, museum teaching, medical field and video gaming entertainment, thanks to the results achieved in a better empathic link between virtual visitor and reconfigured spaces digitally, surpassing in fact, in fluidity of execution and ease of use, any other system of interaction with the simulated environments. It therefore outlines the importance of deepening the benefits and the criticalities associated with the use of such tools in order to improve their usability and expand their use in increasingly common areas. Immersion, presence and interactivity, often as a result of each other and determinants of the quality of a virtual environment, are the peculiarities that define synthetically the particular functionality of the system and in a more general sense are identified in the

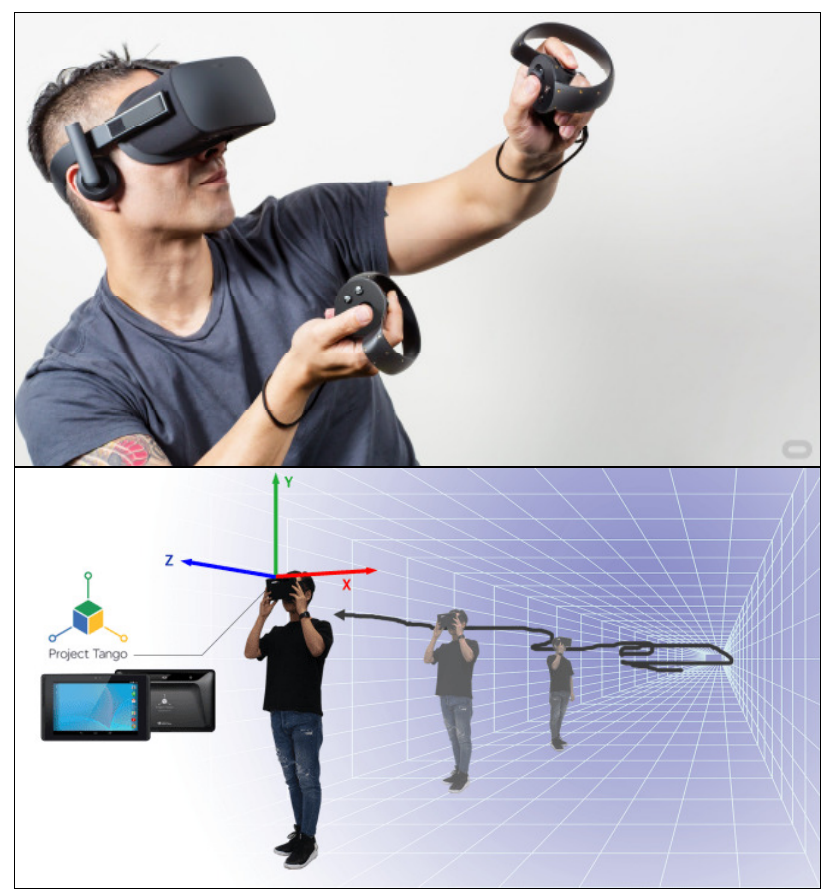

Figure 2. New virtual reality systems applied by and motion tracks, Oculus Rift headset and Project Tango computing platform. Images from www.interactivearchitecture.org/

advances of the Third Digital Revolution (Gershenfeld, 2012), in progress in recent years, which it has quickly gone from CAD vector representation to a three-dimensional reconfiguration manipulated on the desktop, to come, thanks to the advances in the algorithms of light phenomena and the reproduction of materials from physically correct behavior, to an extremely photorealistic representation type, to allow for vivid perceptual exploration and holistic intervention directly on digital assets by means of stereoscopic viewers and their extensions, such as manipulators, joypads, aptic suits etc. Interactivity is still a peculiarity that has always shaped the architectural space in the real world in relation to its functional distribution and use that the environment is capable of offering to the user, defining "architecture" only in terms of usability, functionality and consequently in terms of interactivity. The interactive environment is therefore not necessarily tied to the issue of current technological progress, but rather to relate to the much broader concept of Architecture in its particular relationship with man, with respect to its activities, habits and movements. Focusing on how interaction with human needs can affect the definition of space allows us to understand how the perceptive and interactive component, even in relation to a connotative virtual environment, can concretely promote such a form of spatiality that is based solely on perception by means of instrumentation, defining it to all effects as Architecture not because it exists in the real dimension but because it "interacts" with the human being. The interaction therefore concerns the ability of a user to act on the digitally reconfigured environment, often acquiring virtual domain capabilities that are related to the ability to override and overcome the time-space limits of the real world: there is the ability to fly moving high speed from one side of the scene to moving and manipulating objects and elements impossible to dislocate in the real world, 
changing atmospheric agents, materials and asset form, and so on. In existing virtual systems, interactivity is inseparable from the technological component that regulates the reaction time of the software to the user's actions, in order not to compromise the realism of the experience and to minimize any disturbance of disorientation. The action of falling into the virtual world at the sensorial level thanks to the use of interfaces, in order to perceive the virtual world as existing, identifies the prerogative of the "Immersion", that is, the ability to fit into a closed virtual environment extraneous to the real dimension: it is a relationship with the VR environment that is not closely dependent on the degree of similarity between virtual reconfiguration and physical world, but how this simulation can be quite realistic to extend over time by blocking external distractions and offering the possibility within the experience of selecting the sensory information offered. The connotative reconfiguration approach, similar to a real spatial or derivative from an original creation, thus generates an illusion of virtual spaces perceived in a totalizing way, as lived in a state of complete cognitive immersion. The subjective influence dictated by the direction of a precalculated kinematic sequence and by the perspective canons of a static vision changes in a dynamic and discontinuous representation, able to simultaneously engage visitors of everything that develops in the scene. In virtual dimension, you need to find the same features as a real environment, allowing the user to intervene not only with the objects in the scenario but even in a design editing event, by modifying the three-dimensional macro components that compose it. This requirement can be considered as the propulsive quality for the development of these systems of representation in the workplace, tools that translate the purely representative purpose to seamlessly intervene on personal creativity and the possibility of spatial metamorphosis according to their own compositional taste. Regarding "Presence", the identifying condition of the virtual dimension, can be related to the illusory feeling of being in a "plausible" place despite the awareness of not being there (Steuer, 1992). Sense of Presence, as defined by industry scholars, is critical to virtual users in order to perceive the sense of being in a clear and concrete environment but at the same time non-existent. Increasing attendance as a psychological phenomenon can offer participants a special subjective experience rather than a purely observational experience such as television or movies. Presence can be characterized in three levels: personal presence refers to a sense of "being there"; social presence connects to the feeling of "being there with others"; and the environmental presence finds existence of the virtual space (http://www.interactivearchitecture.org/sense-ofpresence-in-social-vr-experience.html). It always makes more use of these new systems that, freed from being considered merely as instruments of pure representation and recreational equipment, today introduced important scientific instruments, from photogrammetry to $3 \mathrm{D}$ real-time rendering, clearing as a digital model of a architecture or a more articulate and complex scenario can be generated for inclusion in a virtual exploration platform, thus generating the "virtual migration" phenomenon, result of a "virtual cloning" operation, which identifies the dual possibility of transfer of users as Avatars and transfer, through a passage of $3 \mathrm{D}$ metric data, copy scenarios from the real world to the virtual one, making a number of advantages and disadvantages related to a confusion of the natural habits. Assuming a Virtual Survey operation as a cognitive intervention of the existing architectural fabric in archaeological, architectural or museum learning, the main prerogative of this alternative action resides in the possibility of non-invasive investigation, resulting from a prior

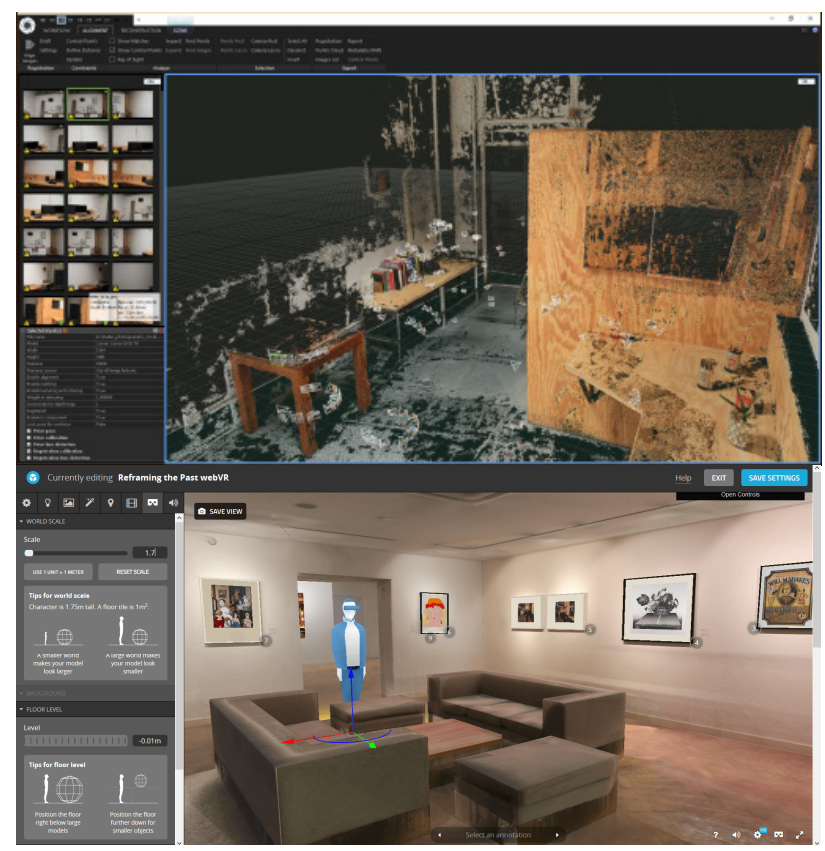

Figure 3. Application of 3d photogrammetry by Reality Capture software and Sketchfab 3d multimedia sharing platform. Images from http://jordanhalsey.com/blog/

acquisition of point cloud data from laser scanners or 3D photogrammetry, together with the opportunity to intervene on spatial modification without affecting the original structure, thus tending to intervene in a cross-fertilization manner, in line with current trends in environmental sustainability and economic savings. Creating and disseminating new low-cost tools such as Microsoft Kinect or new dual-chamber smartphones with RGBD depth sensor (Lee \& Medioni, 2014) has triggered a pricecutting process not only of software professional pads such as Pix4D mapper or Agisoft PhotoScan but also articulated as Drones and Laser Scanners, up to a few years ago very expensive instrumentation and certainly not within reach. The other major factor influencing lowering costs is the E-Learning teaching and migration of skills that exploit cyberspace as a territory through which it is possible to get home from time to time and without space-time constraints at every location, accessing external and client-based utilities and offering services to which access can be accessed directly through the exclusive use of the virtual devices (Roca et al., 2006). This trend is certainly a benefit, both in terms of job simplification and in terms of the development of new forms of work, which are aimed at the establishment of parallel architectural digital structures such as specific programmers, modelers, virtual architects or even archaeologists specialized in the analysis and study of cloned artifacts on the computer. Thanks to cheap software and viewers, the virtual dimension can be transferred to a virtual dimension that can only be accessed through devices, adhering to an operation that, in the present, still carries a series of doubts and criticalities not only tied to technical ones imperfections that are undergoing revision and correction, the main causes of secondary physical disorders associated with intensive use of the equipment such as disorientation or ocularmotor problems, such as Eye-Strain, but also cause much more severe physical damage to blood circulation due to static condition and mental problems of social isolation and dependence. The lack of long term experimentation of such devices, calibrated according to biotipology and age range, could create a dual intervention scenario, on the one hand, limiting the distribution of such instruments to an exclusive use 
of a specialized public, hoping to maintain medium-to-high cost to allow for a longer period of review and testing on a few subjects, on the other hand, assuming a much more profitable, albeit more risky, mass spread of products to in order to be able to test in a short time and for millions of users, benefits and dangers associated with their intensive use. To date, it seems that the trend is projected towards the second option and as a result of the crowd funding experimentation and production of the first low-cost HMD optical viewer, the Oculus Rift, the question of the spread of such low cost systems and in parallel their massive short-term experimentation.

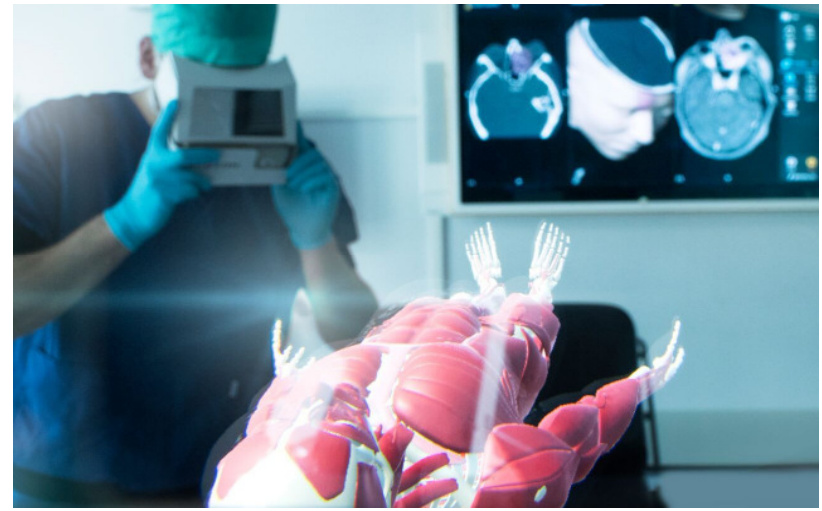

Figure 4. Use of AR headset in the medical-educational area

\section{IMPLEMENTATION WORKFLOW OF SPACE VIRTUALIZATION, EVOLUTION OF METHODOLOGY AND EVALUATION OF RESULTS AND POTENTIAL}

In order to be generated and then transferred to computerized platforms for a virtual immersive tour, the composing elements of a real-world clone scenario require a precise workflow aimed at a fluid and natural interactive exploration, requiring careful planning on some process complexes and concatenate elaborating phases. Knowing and hence adopting a thought-out methodology greatly simplifies the three-dimensional modeling approach set to optimize the export of geometric assets to VR equipment, reducing a number of issues that tend to slow down the work pipeline and in some cases to block the workflow, thus causing continuous corrections in the previous phases. Basically, the work phases can be divided into two main implementation processes, which we call "Cloning from Real" and "Virtual Transfer", both designed to obtain connotative and usable scenarios reconfigured on the exact form of those actually existing. In relation to the first phase, it is undoubtedly a substantial involvement of those new high-tech techniques offered by low-cost instrumentation, such as photomodeling using Structure From Motion (SFM) algorithm, allow us today to dramatically review the surfaces and shape of artifacts of variable magnitude, recording also the material and proportional consistency and the color component. The current methodology also introduces the use of 3D online archives, in order to collect and make possible the sharing of the resulting models, thus enabling more immediate use of information in the form of multimedia inserts to accompany the assets. The use of economically accessible tools, along with the use of software that in the course of a few years have improved the management of the SFM algorithm by achieving a high scientific maturity to achieve results with a high degree of precision, comparable to those achieved by active sensors such as laser scanners offer versatile photomodeling tools designed to conform threedimensional models that can be easily and directly measured. When rudeness, archeological objects and small or medium size fragments are detected, automated photogrammetry is particularly effective, provided that the object to be detected has no reflective surface and that the light exposure is homogeneous. In line with the previous three-dimensional photogrammetric approach, urban-scale elements, which are considerably larger, require the use of drones, even lower-cost ones, in order to obtain a better result in the acquisition of photographic data that involve the structure analyzed in totality. The procedure consists in capturing a series of photographs taken from different points of view to cover the entire surface area of the subject, ensuring an adequate overlap between the frames of the same trajectory. The software thus automatically determines the spatial orientation of each single click to quantify its functional parameters to determine the camera's position with respect to the nodal center shot in the photographic shot, involving the recognition of raster pixels coupled with a small detail of the framed subject. Following the orientation of the shots, the subsequent collimation of the homologous points precisely identifies the projective beam with respect to the node center, generating the cloud point that already computes metric proportions, composition and shape, and identifying the form of the cloned object. The program then interprets the points of the cloud as triangular mesh vertices of a polygonal pattern on which the color component deriving from photographic data is projected. From point cloud to mesh calculation it is possible to intervene on the polygonal density based on the desired visual quality, but also in relation to the required scale requirement. However, there is no adequate geometric interpretation of the clone object by the software that the program simply discretizes as a form generated by a series of interpolated surfaces. At this stage, therefore, the occurrence of artifacts or defects of definition is very common due to the complexity of the scenario, but often to the digital disorder, such as vegetation, which in the photographic repetitions tends to cover part of the structure, generating in fact gaps and imperfections which make the $3 \mathrm{~d}$ model unsuitable for the next phase.

Among the advantages of photomodelling is the ability to use cheap camcorders, including smartphones, with non-calibrated lenses: the new software dedicated to the purpose is, in fact, preset to automatically search the calibration parameters of radial distortion during the analysis phase, thus optimizing the search for homologous points between the images, as it has long been in the sewing process of the sequences, simply with the only means of blocking exposure to white balance in order to obtain a uniformity of the luminous component. It is also necessary to avoid "digital noise" on images by adjusting ISO sensitivity and, alternatively, positioning the sensor at the lowest possible level. A number of correction algorithms are already present in professional products like Agisoft Photoscan but as many open source and low-cost products can offer the same benefits as MeshLab (www.meshlab.net/) and Autodesk Recap 360 (www.autodesk.com/education/free-software/recappro) for fast and dynamic control over polygonal surfaces with criticality. The last phase involves the application, through a projective system, of textures on 3D models created, retopologized and equipped with UV Map, taking advantage of photos already used for photomodelling. A Diffuse map and a Normal map are then generated, requiring a detailed and photorealistic output of the three-dimensional model obtained, exportable and implementable on external rendering platforms of real time rendering (RTR). The cloning phase is not completed by obtaining a visually perfect 3D geometric model, functionally suited for insertion on a digital platform for real time exploration, because are essential particular requirements which involve some of the features inherent in the model itself, such as a low polygonal quantity, an organized geometric mesh 
topology, facing out normals, and a possible use of polygonal density subdivision surfaces on multiple models of the same subject to apply the so-called LOD (Level of Detail) useful for RTR platforms for optimizing calculations and rendering. In this regard, it is advisable to introduce the use of sculpting programs such as 3d Coat (https://3dcoat.com/home/ ) and zbrush (http://pixologic.com/ ), currently very much used for cinematic and artistic productions, but paradoxically at a relatively affordable cost, especially for academic and nonprofit projects, which allow not only to intervene on the morphology of the digital object, allowing the possibility of modifying the internal mesh by means of automatic re-topology systems, but they also offer the option of using fluid digital painting tools, UV mapping tools, adding detail through the creation of Displacement, Normal and Bump maps, polygonal optimization and self-control and fixing surface problems. The Virtual Transfer phase, on the other hand, includes those processes relating to a semantic division of the compositional elements of the total scenario so that it can control the quality of the polygonal mesh and its density with respect to each single fragment. The elements will then be imported, based on the aesthetic rendering features of the rendering engine, on the desired RTR virtual platform, such as Unity, Unreal Engine 4 and Cry Engine, for a couple of years completely free for personal, hobby and search. The management of the simulated illumination effects and the graphic rendering of the physically correct materials has undergone a remarkable technical implementation for this type of application, especially Unreal Engine 4 has achieved photorealism levels very similar to those of the precalculated rendering, such as V-Ray and Arnold Render. According to the latest Vision VR / AR 2017, the worldwide conference that has been held in Hollywood for some years now on the advancement of virtual systems in the graphics computer, is also anticipating the implementation within the software of one of the unbiased GPU rendering engines Otoy Octane Render / Otoy Brigade, capable of using a simultaneous Path Tracing formula, a Global Illumination assimilation algorithm that even shadowy parts retain minimal amount of reflected light and widespread, further enhancing realistic photo rendering and getting real-time visual scenes that really match the reality, with quick calculations and no optimizations. Unreal today uses other alternative systems to improve light simulation, such as the Ray Traced Distance Field and Field Ambient Occlusion, useful tools to soften the net shadows and thus simulate the rather large and near-light sources, and Lightmass bake texturing, through which it is easy to break down the calculation times and to properly solve hardware performance issues. These graphic escamotage exploit properties related to the geometric data of scenes imported from other programs to effectively calculate shadows and bring them to the mesh. During the "Shadowing" phase, a shadow-shade phase projected by a parallel or point light source, only the surfaces intercepted by the light beam are considered, ignoring the calculation for all other parts of the $3 \mathrm{~d}$ model. The shadow edges softness, however, depends on the distance from the radiant element and it is possible to calibrate the quality of the same by increasing or reducing the number of interceptor beams, involving only light-affected pixels. The limits of this technique reside in the same morphology of the model to be shaded: if a mesh is not uniform or affected by tasseling it can generate artifacts in the shadow areas. Lastly, the operational pipeline provides support for a low-cost hmd viewer, thought of VR Homido V2, to allow interactive exploration of architectural scenarios, and the implementation of a specific hardware for an AR experience for the archeological artifact in question, Aryzon (www.kickstarter.com/projects/aryzon/aryzon-3d-augmentedreality-for-every-smartphone), AR apparatus and Mixing
Reality similar to the much more expensive Microsoft HoloLens, in kickstart development crowdfunding just as a few years ago the Oculus Rift, it proves to be an excellent low-cost alternative system that offers performance that can be attributed to virtual reality and that increased with a simple way of using it. In case studies, this executive workflow was followed to complete the cloning phase from the real and subsequent virtual transfer using all the graphical techniques described above to maximize visual and interactive experience without any particular technical problems.

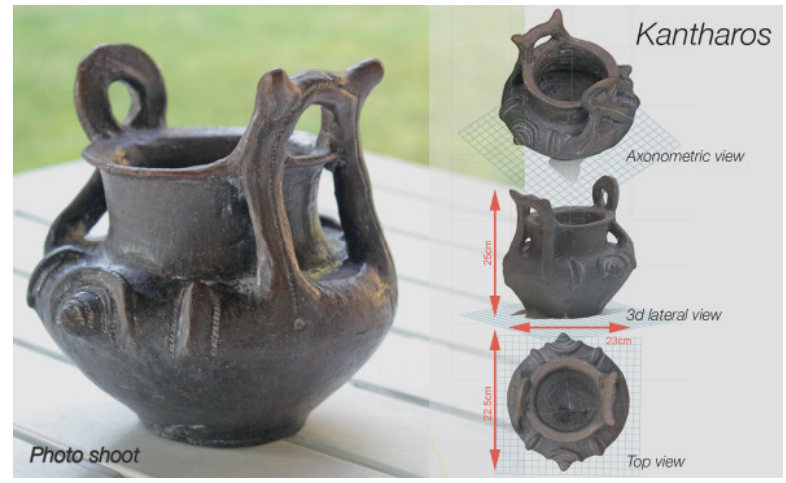

Figure 5. Photographic shots from which the Kantharos polygonal 3D model was obtained using the Recap Photo

\subsection{From the survey to virtual reconstruction for immersion navigation, two case studies compared.}

The research in question focuses on the dynamic experimentation of low-cost instrumentation and hardware aimed at obtaining digital models derived from reality and subsequently explored through virtual reality interfaces. In this regard, a specific methodology has been used that allowed both cloning and virtual transfer on two case studies from apparently dissimilar purposes, one belonging to the archaeological field, introducing the involvement of increased reality in museum teaching, and the other related to the more specific field of architecture, aimed at obtaining an immersive virtual tour compatible with documenting and enhancing the cultural and historical heritage. The first case concerns the $3 \mathrm{~d}$ photogrammetric survey of a small Etruscan Kantharos $(25 \times 23 \mathrm{~cm})$ of the sixth century. B.C, for the sake of accuracy the experiment was carried out on a certified copy of the artifact, currently stored in the warehouses of the archaeological museum of Viterbo. The second relates to the reconstruction of the pronaos of the building of the Aurum, an ex-liquor industry in Pescara, redesigned by Giovanni Michelucci in 1940 on a pre-existence of the early twentieth century whose structure is preserved.

In both study cases we have used the modeling photo algorithms provided by the AUTODESK Recap Photo program, the current evolution of the previous $123 \mathrm{~d}$ catch, always of the same manufacturer, which is greatly improved both in the data management and in the final processing of the $3 \mathrm{~d}$ digital model. The mesh correction functions, holes and gaps, polygonal optimization and retopology have been greatly enhanced. An export mode to the .obj Quod format has also been added, where the $3 \mathrm{~d}$ assets generated in triangular mesh are transformed into quadrangular mesh polygons, with obvious improvement of the geometric shape. The software can leverage both local cpu and gpu internal computing and the on-line remote cloud computing power derived from the sophisticated Autodesk servers. 
On average, a detailed model can take from 2 to 5 hours of processing. Compared to the much more popular but also cheaper Agisoft PhotoScan, which offers much more functionality and the ability to work on all stages of the work, from the alignment of the photos to the creation of the photo frames projected on the mesh, Recap after uploading the photos, it starts the processing in the background and, even if it offers excellent manipulation tools and $3 \mathrm{~d}$ corrective postproduction, it returns the complete polygonal model directly, avoiding any action on the individual phases. The model of the Etruscan ceramic, for example, digitally defined with the software, is in fact unexpectedly well-structured and has detailed textures of good quality, color rendering and resolution, even to a close visual analysis. In relation to the first case study, compatible with a proposal for museum teaching, we supposed the use of Aryzon AR, a new headset with a price of about $\$ 30$ specific for a reality-enhancing experience similar to that offered by the most expensive Microsoft HoloLens. The $3 \mathrm{~d}$ objects were then transferred to the Unreal Engine 4 / Unity virtual platform to be then potentially converted into a compatible format supported by the Aryzon App on smartphones, through which you can finally load the assets accessible by the viewer. The new Dutch company Aryzon hopes to create a passionate content development community for $\mathrm{AR}$, enabling it to optimize and improve its platform in order to become a leader in the creation and sharing of materials compatible with AR.

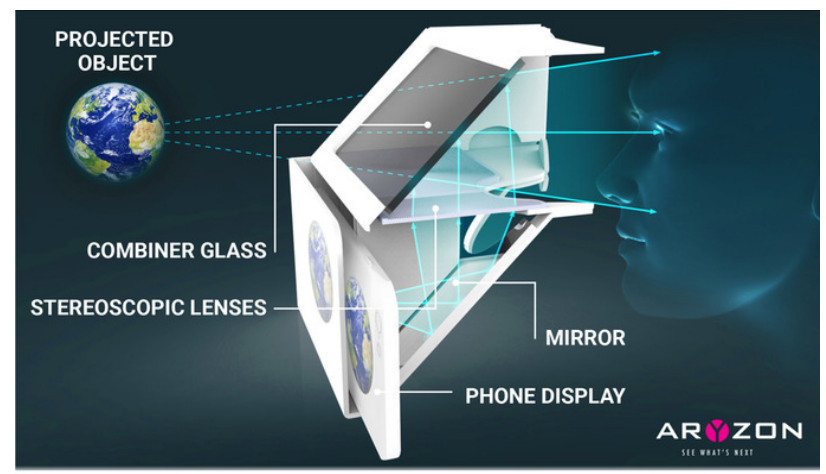

Figure 6. Aryzon 3d operating scheme.

The processing of the second case study on the photogrammetric survey of the double height portico, a total of approximately $12.80 \mathrm{~m}$, has constituted some difficulties in detecting considerably larger entity through low cost instrumentation. For the work has been employed an inexpensive drone mounted with a default hd camera, in order to get shots of the entire facade. Despite the availability of a large number of frames, it was desirable in the processing phase to select only the best shots due to another big limitation of the photogrammetric software used: the Autodesk product allows to upload, in its educational and free version, to maximum 300 frames and does not have any multiple chunk merging capabilities (Palestini \& Basso, 2017). This conditioning can be overcome by using another program, which is really inexpensive compared to its potential, namely Pixologic Zbrush. In the pipeline is an essential tool for the management of major multiple sessions conducted previously on Recap, using functionality similar to that chunk of PhotoScan: although the software does not support point clouds, thanks to a proprietary algorithm it is able to manage billions of polygons with ease, allowing the ability to merge into a single scene, through dynamic Boolean unions, multiple and complex $3 \mathrm{~d}$ portions resulting from multiple sessions of survey on the same subject. Functional to this methodology are also very useful the software self-retopology and remeshing algorithms, which enable a quick mesh management without altering its actual geometry. As in the previous case study, the next phase involves the transfer of $3 \mathrm{~d}$ assets to the Unreal platform.

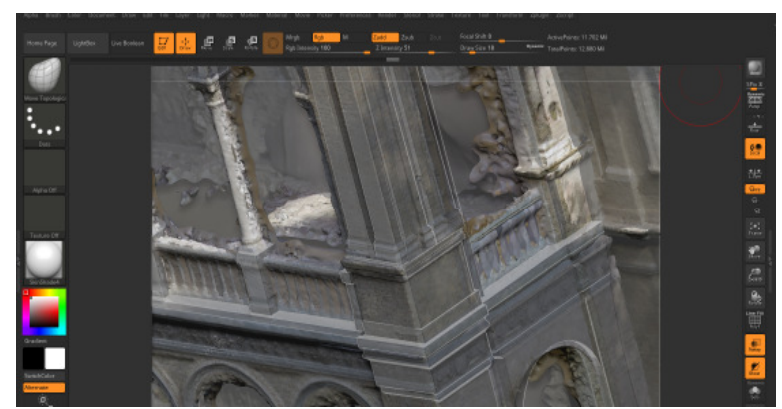

Figure 7. Remodeling process of the semantic elements using zbrush software.

The model, after being adequately triangulated, is then imported through multiple sessions in FBX format, divided into specific semantic elements of no more than 75,000 polygons, and then re-assembled by virtue of the original spatial coordinates. In relation to the real-time rendering of scene lighting, the effects of light and shadow are directly impacted on the textures, in order not to be recalculated to each frame of movement. Thanks to this Baking texture function for Lightmass, also known as "Build Light," which may require, depending on the size and complexity of the $3 \mathrm{~d}$ asset, also some hours of calculation, the light samples are generated in a specific portion of the model, calculated in all the refractions and reflections, so that they can be imprinted directly on the texture of the same. Hardware will then dedicate itself to the calculation of the Ray-tracing algorithm, much faster, exploiting the potential of the graphics card. The experiment carried out on the Unreal Engine has given excellent visual results, although the compositing and light management phases, despite the very clear and intuitive technical interface, were still a little tedious and the system nodes, used by the program to manage mapping, texturing, and interactions, requires at least a basic programming preparation and cannot yet be within reach of everyone. About the implementation of alternative systems to the most expensive Oculus Rift, it is thought to use VR systems that are based on the processors, gyros and movement sensors of the smartphone contained within the viewer itself. In order to limit spending as much as possible using the smartphone lcd screen and sensors, these tools are still in an embryonic development phase of hardware and VR software capabilities, although they have an interesting potential and provide the average consumer with a foretaste of the immersive VR experience. Today they are readily available many not interactive video $360^{\circ}$ on you tube, video games and immersive virtual experiences dedicated to users of mass, downloadable for free on iOS App Store and Google Play. At the conclusion of the pipeline, the test on the use of the low-cost Homido V2 VR visitor has provided good results. The system offers the ability to move without the clutter of external wires and accessories, as is the case with the professional equipment and today even better performance, such as the Oculus Rift, with which a part of the work has been tested. French society, in an attempt to distinguish itself from similar products deriving from the far East, such as Korea and China ${ }^{1}$, offers the product at a very competitive price, just under

${ }^{1}$ The Chinese multinational Xiaomi will soon market Pacific the $\$ 200$ new viewer working without the support of the smartphone. www.bloomberg.com/news/articles/2017-0713/facebook-said-to-plan-200-wireless-oculus-vr-headsetfor-2018 
$£ 50$ in the United Kingdom, offering good-quality adjustable lenses and a 100-degree comfortable viewing range, incredibly ample, with minimal blur, which also boasts alternative settings for the wearers of glasses. This viewer, perhaps not the least expensive, however, offers the most complete and immersive VR experience than any alternative lowcost headset currently on the market.

In the virtual exploration of the Aurum, the textures are perceived correctly, the frame rate is generally kept in the range of 30 to 60 frames per second; the flickering problems are limited in overall visualization, especially when it is approaching too much to $3 \mathrm{~d}$ assets. The system therefore responds quite well to the head movements and positioning of the optical cone with the motion sensor, while as a negative note it can be seen that turning the view too fast and excessive skewing of the head can result in a loss of signal video, this behavior may be due to the calibration not performed correctly according to the scene scale. Basically everything works well enough and from the last two releases to connect a virtual device to the PC on which the Unreal Engine is installed has also become simpler and less intricate. The final step is the compression and export of the Case Study scenario on the most common portable external platforms compatible with Android systems, windows, mac and playstation 4 , designed for direct use on low-end headset that support the internal use of smartphone.

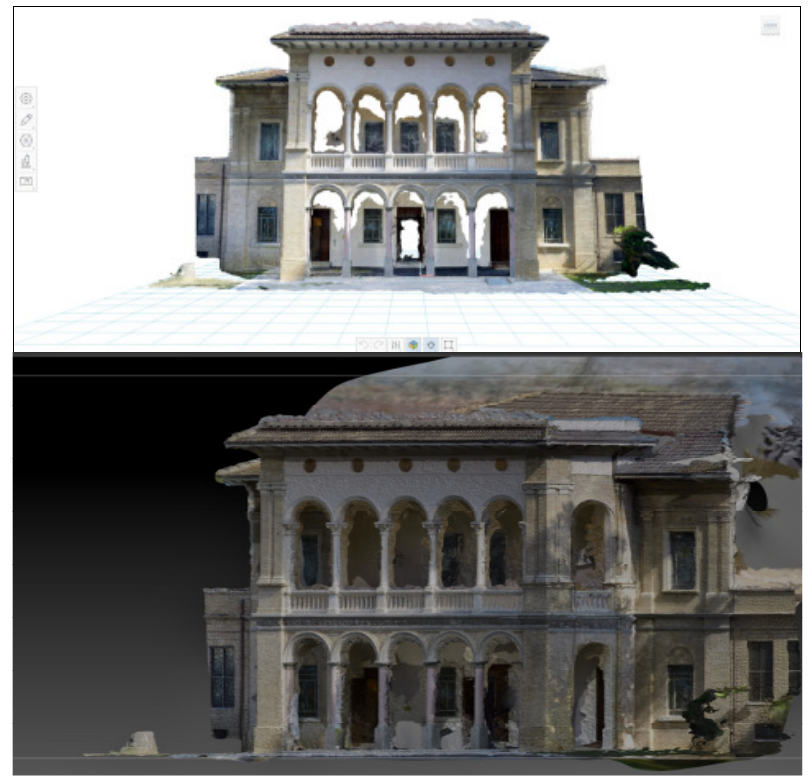

Figure 8. Digital model of the artifact obtained after point cloud computing by Recap Photo

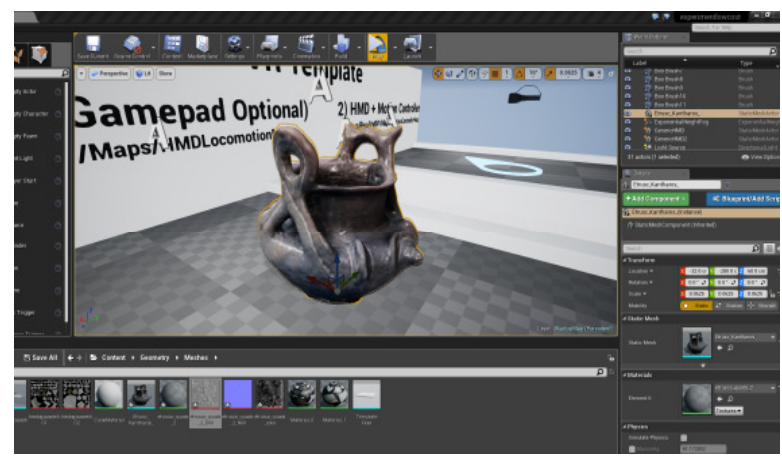

Figure 9. Virtual transfer on Unreal Engine 4.15 platform for immersive tour

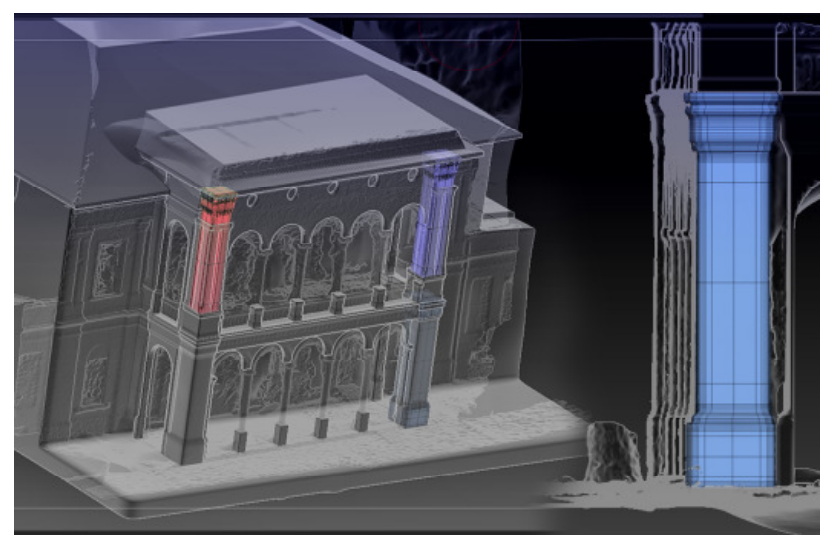

Figure 10. Polygonal model obtained from cloud computing in Recap Photo

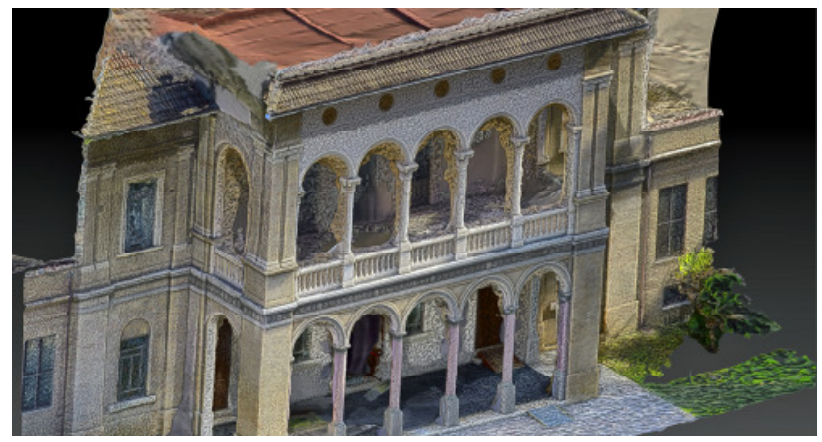

Figure 11. Phases of cleaning up of digital noise and semantic division of the $3 \mathrm{~d}$ assets for the retopology in Zbrush

\section{CONCLUSIONS}

In conclusion, based on the diffusion of new low-cost systems and software, the cloning methodology from the real and the subsequent transfer to virtual platforms now offer greater opportunities for cultural disclosure, better tools of survey and documentation of the current status, a more widespread use in a professional and generally approaching man to the most appropriate technology to improve the quality of life. Low cost software and hardware, compared to their paid counterpart, are becoming more and more powerful and easy to use, gaining economic results from massive sales of devices and distribution apps and upgrade plug-ins that provide extra functionality and additional improvements. In summary, the methodological considerations regarding the choice of these new space virtualization tools to achieve their goals, with the awareness that every purpose has specific prerogatives, such systems used with the right caution, can be a viable alternative within the state of the art investigating the application of technology to support $3 \mathrm{~d}$ vision, making it somewhat more accessible and consequently more comprehensive, thanks to interactive exploration, information and data in the past related to specific cultural and professional fields. 


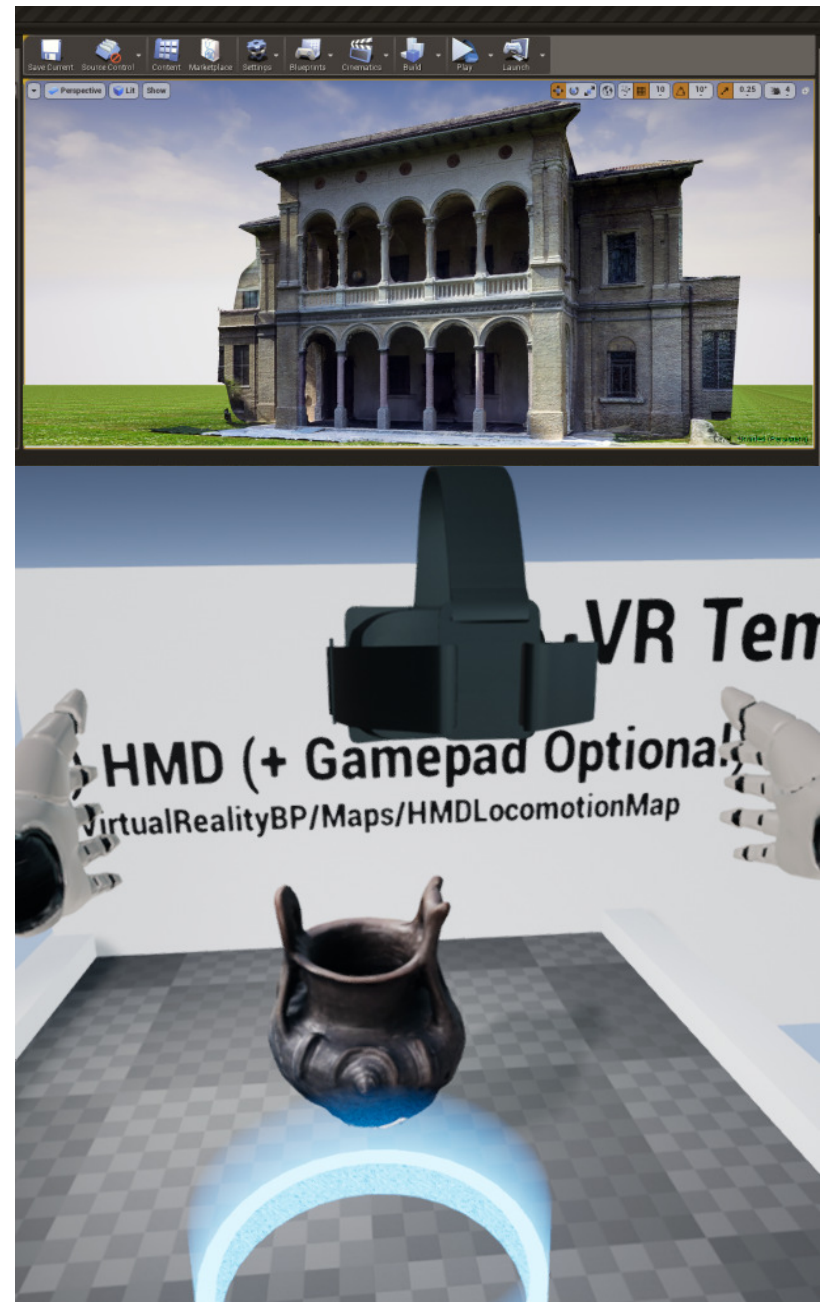

Figure 12. Comparison between the two virtual systems conducted on case studies using the Unreal Engine platform, immersive tour of Aurum building (top) and virtual manipulation on archaeological artefacts (bottom)

\section{ACKNOWLEDGEMENTS}

The authors wish to thank in particular the people involved in the acquisition and management of data and in the project on site among which the architect Matteo Spinozzi for the acquisition of image based data and for the shootings on drone. Also thanks Adriana Gandolfi for having provided the archaeological artefact object of the first case study.

\section{REFERENCES}

Amadesi, E., 1975, Fotointerpretazione e Aerofotogrammetria. Pitagora Ed, Bologna, Italia.

Argelaguet, F., Trico, M., Hoyet, L. and Lecuyer, A., 2016. The role of interaction in virtual embodiment: effects of the virtual hand representation. Proceeding of IEEE Virtual Reality Conference. Greenville, South Carolina, USA, pp. 3-10.

Palestini, C. and Basso, A., 2017. Geomatics as a Survey tool to document and enhance the cultural and landscaped Heritage of the monumental complexes in the montains of Abruzzo. The International Archives of the Photogrammetry, Remote Sensing \& Spatial Information Sciences, 42.
Bennett, A.L., 2008. The Potential and Uniqueness of Virtual Environments for Education. New Horizons in Adult Education and Human Resource Development. 22(3), pp. 53-59.

Casaneuva, J. S., 2001. Presence and co-presence in collaborative virtual environments. Doctoral dissertation, University of Cape Town, Southafrica https://open.uct.ac.za/handle/11427/6383, access 17 July 2017.

De Luca, L., 2011. La Fotomodellazione Architettonica. Flaccovio D. Editore, Palermo, Italia.

Gershenfeld, N., 2012. How to make almost anything: The digital fabrication revolution. Foreign Aff., 91, p. 43.

Held, R. and Durlach, N., 1992. Telepresence. Presence: Teleoperators and Virtual Environments, 1(1), pp. 109-112.

Iwata, H., 1999. Walking About on an Infinite Floor, Proc. of Virtual Reality '99. March 13-17, Houston, Texas, IEEE Computer Society Press, 286-293.

Lee, Y. H. and \& Medioni, G., 2014. Wearable RGBD indoor navigation system for the blind. European Conference on Computer Vision, pp. 493-508.

McLellan, H., 2001. Virtual Reality. New York: McLellan Wyatt Digital.

Metz, R., 2015. Inside the First VR Theme Park. Report MIT Technology Review. https://www.technologyreview.com/s/ 544096/inside-the-first-vr-theme-park/, access 18 May 2017.

Moskaliuk, J. and Kimmerle, J., 2011. Knowledge Building in User-Generated Online Virtual Realities. Journal of Emerging Technologies in Web Intelligence, 3(1), pp.38-46.

Roca, J. C., Chiu, C. M. and Martínez, F. J., 2006. Understanding e-learning continuance intention: An extension of the Technology Acceptance Model. International Journal of human-computer studies, 64(8), pp. 683-696.

Schuemie, M. J. and van der Mast, C. A. P. G., 1999. Presence: Interacting in virtual reality. Proceedings, Twente Workshop on Language Technology, 15, pp. 213-217.

Steuer, J., 1992. Defining Virtual Reality: Dimensions Determining Telepresence. Journal of Communication, 42(4), 73-93. 